\title{
OSCILLATION WITH RESPECT TO PARTIAL VARIABLES OF LINEAR SECOND-ORDER DIFFERENTIAL SYSTEMS
}

\author{
ZONGQI DENG AND SHIGUI RUAN
}

(Communicated by Kenneth R. Meyer)

\begin{abstract}
Consider the second-order vector differential system (1) $x^{\prime \prime}(t)+$ $Q(t) x(t)=0$ and matrix differential system (2) $X^{\prime \prime}(t)+Q(t) X(t)=0$, where $x(t)$ is an $n$-dimensional vector function and $X(t)$ and $Q(t)$ are $n \times n$ continuous matrix functions. In this article, we establish the concept that systems (1) and (2) are oscillatory with respect to partial variables. Some sufficient conditions are obtained; several examples are given to illustrate the results.
\end{abstract}

\section{INTRODUCTION}

Consider the second-order vector differential system

$$
x^{\prime \prime}(t)+Q(t) x(t)=0
$$

where $Q(t)=\left(q_{i j}(t)\right)$ is an $n \times n$ continuous matrix function on $[a, \infty)$ and $x(t)$ is an $n$-dimensional vector function. Some properties of (1) such as oscillation, disconjugacy, and Sturm comparison theory have been studied by many authors (see $[1-4,6,8-11]$ and the references contained therein).

We will call system (1) oscillatory on $[a, \infty)$, if for $T \geq a$, there exist $\alpha$, $\beta \geq T$ and a nontrivial vector solution $x(t)$ of (1) satisfying $x(\alpha)=x(\beta)=0$, i.e., nontrivial vector solution $x(t)$ of $(1)$ has arbitrarily large zeros on $[a, \infty)$. In this case, obviously every component $x_{i}(t) \quad(1 \leq i \leq n)$ of the vector $x(t)$ has arbitrarily large zeros on $[a, \infty)$.

Example 1. Consider the following system:

$$
\left(\begin{array}{l}
x_{1} \\
x_{2} \\
x_{3}
\end{array}\right)^{\prime \prime}+\left(\begin{array}{ccc}
1 & 0 & 0 \\
1 & -1 & 0 \\
1 & 0 & 0
\end{array}\right)\left(\begin{array}{l}
x_{1} \\
x_{2} \\
x_{3}
\end{array}\right)=\left(\begin{array}{l}
0 \\
0 \\
0
\end{array}\right) .
$$

Received by the editors April 28, 1990 and, in revised form, August 4, 1990.

1980 Mathematics Subject Classification (1985 Revision). Primary 34A30; Secondary 34C10.

Key words and phrases. Oscillation with respect to partial variable, prepared solutions, secondorder differential systems. 
It is easy to see that the linearly independent nontrivial solutions of (2) are

$$
\begin{array}{lll}
X_{11}=\left(\begin{array}{c}
\sin t \\
\frac{1}{2} \sin t \\
\sin t
\end{array}\right), & X_{12}=\left(\begin{array}{c}
\sin t \\
e^{t}+\frac{1}{2} \sin t \\
t+\sin t
\end{array}\right), & X_{13}=\left(\begin{array}{c}
\sin t \\
e^{t}+\frac{1}{2} \sin t \\
1+\sin t
\end{array}\right) \\
X_{21}=\left(\begin{array}{c}
\cos t \\
\frac{1}{2} \cos t \\
\cos t
\end{array}\right), & X_{22}=\left(\begin{array}{c}
\cos t \\
e^{t}+\frac{1}{2} \cos t \\
t+\cos t
\end{array}\right), & X_{23}=\left(\begin{array}{c}
\cos t \\
e^{t}+\frac{1}{2} \cos t \\
1+\cos t
\end{array}\right) .
\end{array}
$$

Obviously, solutions $X_{11}$ and $X_{21}$ are oscillatory, that is, all components of $X_{11}$ and $X_{21}$ have arbitrarily large zeros on $[a, \infty)$, but just the first and the third components of $X_{13}$ and $X_{23}$ have arbitrarily large zeros on $[a, \infty)$, and only the first components of $X_{12}$ and $X_{22}$ have arbitrarily large zeros.

The above example indicates that when we study the oscillation of system (1), we will meet the special case that only some of the components (variables) of nontrivial solutions $x(t)$ have arbitrarily large zeros on $[a, \infty)$.

We establish the following concept:

Definition 1. For a nontrivial vector solution $x(t)=\operatorname{col}\left(x_{1}(t), x_{2}(t), \ldots, x_{n}(t)\right)$ of system (1), if only $k(1 \leq k \leq n)$ components of $x(t)$ have arbitrarily large zeros on $[a, \infty)$, then we say that $x(t)$ is oscillatory with respect to the $k$ partial variables. If all nontrivial vector solutions of (1) are oscillatory with respect to the same $k$ variables, then we say that system (1) is oscillatory with respect to the $k$ partial variables.

Remark 1. We do not require that the $k$ components of $x(t)$ vanish at the same points. For example, both the first component $x_{1}=\sin t$ and the third component $x_{3}=1+\sin t$ of solution $X_{13}$ have arbitrarily large zeros on $[0, \infty)$; we say that $X_{13}$ is oscillatory with respect to partial variables $x_{1}=\sin t$ and $x_{2}=1+\sin t$, but $x_{1}=\sin t$ and $x_{2}=1+\sin t$ do not have the same zeros on $[0, \infty)$. Similarly, solution $X_{23}$ is oscillatory with respect to the partial variables $x_{1}=\cos t$ and $x_{3}=1+\cos t$. And every solution $X_{i j} \quad(i=1,2$, $j=1,2,3)$ of system (2) is oscillatory with respect to its first component $x_{1}(t)$; hence system $(2)$ is oscillatory with respect to the partial variable $x_{1}(t)$.

For convenience, we suppose that the $k$ partial variables are the first $k$ components of $x(t)$, i.e., we only study the oscillation with respect to partial variables $y(t)=\operatorname{col}\left(x_{1}(t), x_{2}(t), \ldots, x_{k}(t)\right)(1 \leq k \leq n)$.

Remark 2. If $k=n$ in Definition 1 , then $x(t)$ is oscillatory with respect to all variables; this is the ordinary case about the oscillation.

Consider the second-order matrix differential system

$$
X^{\prime \prime}(t)+Q(t) X(t)=0
$$

in which $X(t), Q(t)$ are $n \times n$ continuous matrix functions on $[a, \infty)$ and $Q(t)$ is symmetric. A matrix solution $X(t)$ of $(3)$ is called a nontrivial solution, if $\operatorname{det} X(t) \neq 0$ for at least one $t \in[a, \infty)$. If a nontrivial solution $X(t)$ of (3) satisfies $X^{T} X^{\prime}=\left(X^{T} X^{\prime}\right)^{T}$, then $X(t)$ is called a prepared solution, where 
$X^{T}$ denotes the transpose of $X$. If for any $b>a$ and for every nontrivial prepared solution $X(t)$ of $(3)$, det $X(t)$ vanishes on $[b, \infty)$, then we say that system (3) is oscillatory.

For system (3) we have the following definition:

Definition 2. Let $X(t)$ be a nontrivial prepared solution of system (3). If for any $b>a$ at least one of the $k$ th order determinants $(1 \leq k \leq n)$ vanishes on $[b, \infty)$, we say that the nontrivial prepared solution $X(t)$ of system $(3)$ is oscillatory with respect to the $k$ partial variables. If every nontrivial prepared solution $X(t)$ of system (3) is oscillatory with respect to the same $k$ partial variables, then we say that system (3) is oscillatory with respect to the $k$ partial variables.

Remark 3. Similarly, we do not require that the $k$ th order determinants vanish at the same points on $[b, \infty)$.

Example 2. Consider the matrix differential system

$$
\left(\begin{array}{lll}
x_{11} & x_{12} & x_{13} \\
x_{21} & x_{22} & x_{23} \\
x_{31} & x_{32} & x_{33}
\end{array}\right)^{\prime \prime}+\left(\begin{array}{ccc}
1 & 0 & 0 \\
1 & -1 & 0 \\
1 & 0 & 0
\end{array}\right)\left(\begin{array}{lll}
x_{11} & x_{12} & x_{13} \\
x_{21} & x_{22} & x_{23} \\
x_{31} & x_{32} & x_{33}
\end{array}\right)=\left(\begin{array}{l}
0 \\
0 \\
0
\end{array}\right) .
$$

We can rewrite matrix system (4) as the following three vector systems

$$
\begin{aligned}
& \left(\begin{array}{l}
x_{11} \\
x_{21} \\
x_{31}
\end{array}\right)^{\prime \prime}+\left(\begin{array}{ccc}
1 & 0 & 0 \\
1 & -1 & 0 \\
1 & 0 & 0
\end{array}\right)\left(\begin{array}{l}
x_{11} \\
x_{21} \\
x_{31}
\end{array}\right)=\left(\begin{array}{l}
0 \\
0 \\
0
\end{array}\right), \\
& \left(\begin{array}{l}
x_{12} \\
x_{22} \\
x_{32}
\end{array}\right)^{\prime \prime}+\left(\begin{array}{ccc}
1 & 0 & 0 \\
1 & -1 & 0 \\
1 & 0 & 0
\end{array}\right)\left(\begin{array}{l}
x_{12} \\
x_{22} \\
x_{32}
\end{array}\right)=\left(\begin{array}{l}
0 \\
0 \\
0
\end{array}\right), \\
& \left(\begin{array}{l}
x_{13} \\
x_{23} \\
x_{33}
\end{array}\right)^{\prime \prime}+\left(\begin{array}{ccc}
1 & 0 & 0 \\
1 & -1 & 0 \\
1 & 0 & 0
\end{array}\right)\left(\begin{array}{l}
x_{13} \\
x_{23} \\
x_{33}
\end{array}\right)=\left(\begin{array}{l}
0 \\
0 \\
0
\end{array}\right) .
\end{aligned}
$$

It is easy to see that matrix system (4) is oscillatory with respect to the partial variables $\left(x_{11}, x_{12}, x_{13}\right)$.

In this paper, we study the oscillations of vector system (1) and matrix system (4) with respect to partial variables. Sufficient conditions are obtained in terms of coefficient matrices of (1) and (4).

Recall that a number $b>a$ is said to be a conjugate point of $a$ if there exists a nontrivial solution $x(t)$ of system (1) satisfying $x(a)=x(b)=0$. System (1) is said to be disconjugate on an interval $[a, b]$ if there are no conjugate points, therein, i.e., every nontrivial solution vanishes at most once in $[a, b]$. 


\section{MAIN RESUlts}

The following result is due to Ahmad [1].

Lemma 1. Let $Q(t)=\left(q_{i j}(t)\right)$ be continuous on $(a, \infty)$ with $q_{i j}(t) \geq 0,1 \leq i$, $j \leq n$. If $(1)$ is disconjugate on $(a, \infty)$, then there exists a nontrivial vector solution $u=\operatorname{col}\left(u_{1}, u_{2}, \ldots, u_{n}\right)$ of (1) such that $u(a)=0$ and $u_{i}(t) \geq 0$ for $i=1,2, \ldots, n$ and $t \geq a$.

Theorem 1. Assume that $Q(t)=\left(q_{i j}(t)\right)$ is continuous on $(a, \infty)$ and $q_{i j}(t) \geq$ $0,1 \leq i, j \leq n$. If there exists an integer $k, 1 \leq k \leq n$, such that each column of the first $k$ columns of $Q(t)$ contains an element $q_{i j}(t)$ such that $\int_{a}^{\infty} q_{i j}(s) d s=\infty$, then system (1) is oscillatory with respect to the $k$ partial variables.

Proof. Let $y(t)=\operatorname{col}\left(x_{1}(t), \ldots, x_{k}(t)\right), z(t)=\operatorname{col}\left(x_{k+1}(t), \ldots, x_{n}(t)\right)$, and let $x(t)=\operatorname{col}(y(t), z(t))$ be a nontrivial vector solution of system (1). Suppose that (1) is not oscillatory with respect to $y(t)$. Then $x(t)$ is nonoscillatory on $[a, \infty)$; hence for some $T>a$, system (1) is disconjugate on $(T, \infty)$. By Lemma 1, there exists a nontrivial solution $u=\operatorname{col}\left(u_{1}, \ldots, u_{n}\right)$ of (1) such that $u_{i}(t) \geq 0$ on $(T, \infty), 1 \leq i \leq n$. Since for each $i, 1 \leq i \leq n$, we have

$$
u_{i}^{\prime \prime}(t)+\sum_{j=1}^{n} q_{i j}(t) u_{j}(t)=0
$$

so $u_{j}^{\prime \prime}(t) \leq 0$ for $t \geq T$. It follows that $u_{i}^{\prime}(t) \geq 0$ for $t \geq T$. For, if $u_{i}^{\prime}(t)<0$ for some $t_{0}>T$, then $u_{i}^{\prime}(t) \leq u_{i}^{\prime}\left(t_{0}\right)<0$ for some $t \geq t_{0}$. But this would imply that $u_{i}(t) \rightarrow-\infty$ as $t \rightarrow-\infty$, in contradiction to the fact that $u_{i}(t) \geq 0$.

Now, since $u_{i}(t) \geq 0$ and $u_{i}^{\prime}(t) \geq 0$ on $(T, \infty)$, it follows that for each $i$, $1 \leq i, j \leq k \leq n$, either $u_{i}(t) \equiv 0$ or $u_{i}(t)>0$ for $t>T$. If $u_{i}(t) \equiv 0$ for all $1 \leq i \leq k$ and all $t>T$, then $u_{1}(t), \ldots, u_{k}(t)$ have arbitrarily large zeros on $[T, \infty)$, that is, $u(t)$ is oscillatory with respect to $u_{1}(t), \ldots, u_{k}(t)$, which contradicts the assumption. So for $T_{0}>T$, there exists an integer $j$, $1 \leq j \leq k \leq n$, such that $u_{j}(t)>0$ for $t>t_{0}$. By hypothesis, there is an integer $i, 1 \leq i \leq k \leq n$, such that

$$
\int_{T_{0}}^{\infty} q_{i j}(s) d s=\infty
$$

Since $u_{j}(t)>0$ and $u_{j}^{\prime}(t) \geq 0$ on $\left(T_{0}, \infty\right)$, we can find a real number $p$ such that $0<p \leq u_{j}(t)$ for $t \geq T_{0}$. If we let $e_{j}$ be the $j$ th unit basis vector, then $p e_{j} \leq u(t)$ for $t \geq T_{0}$. Therefore

$$
p Q(t) e_{j}+u^{\prime \prime}(t) \leq Q(t) u(t)+u^{\prime \prime}(t)=0,
$$

from which it follows that $p q_{i j}(t) \leq-u_{i}^{\prime \prime}(t)$ and consequently that

$$
P \int_{T_{0}}^{T} q_{i j}(s) d s-u_{j}^{\prime}\left(T_{0}\right) \leq-u_{i}^{\prime}(t) .
$$


But from (5), it follows that $u_{i}^{\prime}(t) \rightarrow-\infty$ as $t \rightarrow-\infty$, in contradiction to the fact that $u_{i}^{\prime}(t) \geq 0$ for $t \geq t_{0}$. This contradiction completes the proof.

Corollary. Suppose there exists an integer $k, 1 \leq k \leq n$, such that $q_{i j}(t) \geq 0$ for $1 \leq i, j \leq k \leq n$, and $\int_{a}^{\infty} q_{i i}(s) d s=\infty$ for each $i, 1 \leq i \leq k \leq n$, then system (1) is oscillatory with respect to the $k$ partial variables.

To discuss the oscillation with respect to partial variables for the matrix system (3), we recall the following notation (cf. [5]): for any $n \times n$ symmetric matrix $Q$, the sequence of symmetric matrices $Q_{k}=\left(q_{i j}\right), i, j=1,2, \ldots, k$ for $k=1,2, \ldots, n$, satisfies $\lambda_{j+1}\left(Q_{k+1}\right) \leq \lambda_{j}\left(Q_{k}\right) \leq \lambda_{j}\left(Q_{j+1}\right)$, where $\lambda_{j}\left(Q_{k}\right)$ denotes the $j$ th eigenvalue of $Q_{k}, 1 \leq j \leq k$, and we take it that $\lambda_{1}\left(Q_{k}\right)$ indicates the largest eigenvalue of $Q_{k}$. The trace of $Q_{k}$ will be designed by $\operatorname{tr}\left(Q_{k}\right)$.

Lemma 2. Let $V(t)=V^{T}(t)$ be an $n \times n$ continuous matrix for $t \geq a$. Suppose there exists an integer $k, 1 \leq k \leq n$, such that for $t \geq a$

$$
\limsup _{t \rightarrow \infty} \frac{1}{t} \operatorname{tr}\left(V_{k}(t)+\int_{a}^{t} V_{k}^{2}(s) d s\right) \leq M<+\infty
$$

for some fixed constant $M>0$. Then it follows that

$$
\liminf _{t \rightarrow \infty} \frac{1}{t} \lambda_{1}\left(\int_{a}^{t} V_{k}^{2}(s) d s\right)<\infty .
$$

The proof is similar to that of Lemma 2.1 in [10] and is omitted.

Theorem 2. Suppose that $Q(t)$ is an $n \times n$ continuous symmetric matrix for $t \geq a$. If there exists an integer $k, 1 \leq k \leq n$, such that

(i) ${ }_{k} \quad \liminf _{t \rightarrow \infty} \frac{1}{t} \operatorname{tr}\left(\int_{a}^{t} Q_{k}(s) d s\right)>-\infty$

(ii) $\lim _{t \rightarrow \infty} \lambda_{1}\left(\int_{a}^{t} Q_{k}(s) d s\right)=\infty$,

then system (3) is oscillatory with respect to the $k$ partial variables.

Proof. On the contrary, we assume that system (3) is nonoscillatory for all variables; then there exists an $a \geq 0$ such that system (3) is disconjugate on $[a, \infty)$. Hence, by [7, p. 388, Theorem 10.2] there exists a nontrivial prepared solution $X(t)$ of (3) such that $\operatorname{det} X(t) \neq 0$ on $(a, \infty)$. Define $V(t)=X^{\prime}(t) X^{-1}(t)$, $t \geq a$. By (3) we obtain the Riccati equation

$$
-V^{\prime}(t)=Q(t)+V^{2}(t), \quad t \geq a .
$$

Since $V(t)=V^{T}(t)$, integrating (8) from $a$ to $t$, we have that for $1 \leq k \leq n$

$$
\begin{aligned}
-V_{k}(t)+V_{k}(a) & =\int_{a}^{t} Q_{k}(s) d s+\int_{a}^{t} V_{k}^{2}(s) d s \\
& \geq \int_{a}^{t} Q_{k}(s) d s, \quad t \geq a
\end{aligned}
$$

then

$$
\lambda_{1}\left(\int_{a}^{t} Q_{k}(s) d s\right) \leq \lambda_{1}\left(-V_{k}(t)\right)+\lambda_{1}\left(V_{k}(a)\right), \quad t \geq a
$$


Taking the limit as $t \rightarrow \infty$ in (11) we obtain $\lim _{t \rightarrow \infty} \lambda_{1}\left(-V_{k}(t)\right)=\infty$, then $\lim _{t \rightarrow \infty} \lambda_{1}\left(V_{k}^{2}(t)\right)=\infty$, hence $\lim _{t \rightarrow \infty} \operatorname{tr}\left(V_{k}^{2}(t)\right)=\infty$. Similar to the procedure in [10], we get

$$
\lim _{t \rightarrow \infty} \frac{1}{t} \lambda_{1}\left(\int_{a}^{t} V_{k}^{2}(s) d s\right)=\infty .
$$

Now taking the trace functional on both sides of (9), we have

$$
\operatorname{tr}\left(V_{k}(t)+\int_{a}^{t} V_{k}^{2}(s) d s\right)=\operatorname{tr}\left(V_{k}(a)\right)-\operatorname{tr}\left(\int_{a}^{t} Q_{k}(s) d s\right) .
$$

So

$$
\begin{aligned}
& \limsup _{t \rightarrow \infty} \frac{1}{t} \operatorname{tr}\left(V_{k}(t)+\int_{a}^{t} V_{k}^{2}(s) d s\right) \\
& \quad \leq \lim _{t \rightarrow \infty} \frac{1}{t} \operatorname{tr}\left(V_{k}(a)\right)-\liminf _{t \rightarrow \infty} \frac{1}{t} \operatorname{tr}\left(\int_{a}^{t} Q_{k}(s) d s\right) \\
& \quad \leq M<+\infty .
\end{aligned}
$$

Lemma 2 now implies that

$$
\liminf _{t \rightarrow \infty} \frac{1}{t} \lambda_{1}\left(\int_{a}^{t} V_{k}^{2}(s) d s\right)<\infty
$$

which contradicts (12) and completes the proof.

\section{EXAMPLES}

Example 3. Consider the second-order vector differential system

$$
\left(\begin{array}{l}
x_{1} \\
x_{2}
\end{array}\right)^{\prime \prime}+\left(\begin{array}{cc}
1 & 0 \\
0 & \frac{1}{4} t^{-2}
\end{array}\right)\left(\begin{array}{l}
x_{1} \\
x_{2}
\end{array}\right)=\left(\begin{array}{l}
0 \\
0
\end{array}\right) \text {. }
$$

The first column of $Q(t)$ contains an element $q_{11}(t)=1$, which satisfies that $\int_{0}^{\infty} q_{11}(s) d s=\infty$. By Theorem 1, system (14) is oscillatory with respect to the partial variable $x_{1}(t)$.

Example 4. Consider the second-order matrix differential system

$$
\left(\begin{array}{llll}
x_{11} & x_{12} & x_{13} & x_{14} \\
x_{21} & x_{22} & x_{23} & x_{24} \\
x_{31} & x_{32} & x_{33} & x_{34} \\
x_{41} & x_{42} & x_{43} & x_{44}
\end{array}\right)^{\prime \prime}+\left(\begin{array}{cccc}
0 & -\frac{3}{2} t^{\frac{1}{2}} & c_{1} & d_{1} \\
-\frac{3}{2} t^{\frac{1}{2}} & -1+\frac{1}{2} t^{-\frac{1}{2}} & c_{2} & d_{2} \\
c_{1} & c_{2} & c_{3} & d_{3} \\
d_{1} & d_{2} & d_{3} & d_{4}
\end{array}\right)\left(\begin{array}{llll}
x_{11} & x_{12} & x_{13} & x_{14} \\
x_{21} & x_{22} & x_{23} & x_{24} \\
x_{31} & x_{32} & x_{33} & x_{34} \\
x_{41} & x_{42} & x_{43} & x_{44}
\end{array}\right)=\left(\begin{array}{l}
0 \\
0 \\
0 \\
0
\end{array}\right),
$$

where $c_{i}=c_{i}(t), d_{j}=d_{j}(t),(i=1,2,3, j=1,2,3,4)$ are arbitrarily continuous functions on $[0, \infty)$ vanishing at $t=0$. With $k=2$ in Theorem 2 , we can calculate that

$$
\begin{gathered}
\int_{0}^{t} Q_{2}(s) d s=\left(\begin{array}{cc}
0 & -t^{\frac{3}{2}} \\
-t^{\frac{3}{2}} & -t+t^{\frac{1}{2}}
\end{array}\right) \\
\lambda_{1}\left(\int_{0}^{t} Q_{2}(s) d s\right)=t^{\frac{1}{2}} \rightarrow \infty, \quad \lambda_{2}\left(\int_{0}^{t} Q_{2}(s) d s\right)=-t,
\end{gathered}
$$


Since

$$
\liminf _{t \rightarrow \infty} \frac{1}{t} \operatorname{tr}\left(\int_{0}^{t} Q_{2}(s) d s\right)=\liminf _{t \rightarrow \infty} \frac{-t+t^{\frac{1}{2}}}{t} \geq-1>-\infty .
$$

By Theorem 2, we know that the above system is oscillatory with respect to the partial variables $y_{1}=\operatorname{col}\left(x_{11}, x_{21}\right)$ and $y_{2}=\operatorname{col}\left(x_{12}, x_{22}\right)$.

\section{ACKNOWLEDGMENT}

The authors would like to thank the referee for making them aware of where [1] was published and sending them a copy of this paper.

\section{REFERENCES}

1. S. Ahmad, On positivity of solutions and conjugate points of nonselfadjoint systems, Bull. Acad. Polon. Sci. Sér. Sci. Math. 27 (1979), 71-75.

2. S. Ahmad and A. C. Lazer, A new generalization of the Sturm comparison theorem to selfadjoint systems, Proc. Amer. Math. Soc. 68 (1978), 185-188.

3. S. Ahmad and C. C. Travis, Oscillation criteria for second order differential systems, Proc. Amer. Math. Soc. 71 (1978), 247-252.

4. F. V. Atkinson, H. G. Kaper, and M. K. Kwong, An oscillation criterion for linear second order differential systems, Proc. Amer. Math. Soc. 94 (1985), 91-96.

5. R. Bellman, Introduction to matrix analysis, 2nd ed., McGraw-Hill, New York, 1970.

6. G. J. Butler, L. H. Erbe, and A. B. Mingarelli, Riccati techniques and variational principles in oscillation theory for linear systems, Trans. Amer. Math. Soc. 303 (1987), 263-282.

7. P. Hartman, Ordinary differential equations, 2nd ed., Birkhäuser, Boston, 1982.

8. W. J. Kim, Comparison theorems for second order differential systems, Proc. Amer. Math. Soc. 96 (1986), 287-293.

9 M. K. Kwong, H. G. Kaper, Z. Akiyama, and A. B. Mingarelli, Oscillation of linear secondorder differential systems, Proc. Amer. Math. Soc. 91 (1984), 85-91.

10. A. B. Mingarelli, On a conjecture for oscillation of second order ordinary differential systems, Proc. Amer. Math. Soc. 82 (1981), 593-598.

11. S. Ruan and Z. Deng, Oscillation criteria for second order linear differential systems, J. Central China Normal Univ. 24 (1990), 1-6.

Department of Mathematics, Huazhong Normal University, Wuhan 430070, Peoples REPUBLIC OF CHINA

Department of Mathematics, University of Saskatchewan, Saskatoon, Canada S7N OW0

Current address: Department of Mathematics, University of Alberta, Edmonton, Canada T6G 2G1 